\title{
Power Quality Experimental Analysis on Rural Home Grid-Connected PV Systems
}

\author{
Rita Jorge Cerqueira Pinto, Sílvio José Pinto Simões Mariano, \\ and Maria do Rosário Alves Calado
}

Instituto de Telecomunicações (IT), Department of Electromechanical Engineering, University of Beira Interior, 6201-001 Covilhã, Portugal

Correspondence should be addressed to Maria do Rosário Alves Calado; rc@ubi.pt

Received 9 March 2015; Revised 25 May 2015; Accepted 9 June 2015

Academic Editor: Wayne A. Anderson

Copyright (C) 2015 Rita Jorge Cerqueira Pinto et al. This is an open access article distributed under the Creative Commons Attribution License, which permits unrestricted use, distribution, and reproduction in any medium, provided the original work is properly cited.

\begin{abstract}
Microgeneration is the small-scale generation of heat or electric power or both, by individuals or buildings to meet their own needs. Recently, microgeneration is being regarded as a means to decentralize the power production of renewable energies, reducing the impacts on the grid caused by unexpected energy demands. Given the increase in microgeneration facilities, determining the quantity of energy produced and the power quality assumes growing importance in low, medium, or high voltage facilities. This paper presents a power quality analysis of two different facilities with photovoltaic generation localized in a rural area of Portugal, describing the voltage and frequency behaviour, the harmonic contents, and the total harmonic distortion. Statistical data are presented regarding the number of voltage events and occurrence of dips and swells in both facilities as a percentage of rated voltage. We conclude that some PV systems can severely affect voltage quality, forcing the grid to work at and even above the maximum voltage standard limit.
\end{abstract}

\section{Introduction}

Increases in fossil fuel prices and growing concerns about global warming are leading the market to seek friendlier ways to generate energy, in particular electricity. Microgeneration can be used for both heat and power and is a viable solution to satisfy residential power/thermal loads while achieving the Kyoto targets, given its good environmental performance, high efficiency, and low emission of harmful greenhouse gases. However, before introducing microgeneration systems, a number of issues should be resolved in terms of system integration, reliability, and safety, particularly concerning the voltage quality in photovoltaic (PV) systems facilities.

Electricity is mostly generated in very large plants, distant from consumer centers. The output is distributed to consumers via a network of high-voltage cables that covers the country, with links to neighboring countries. Substantial energy is lost in the delivery system and Portugal, in particular, has losses in transport and distribution of around $9 \%$ of total electricity generated [1].
In developed countries, houses and services together constitute the major consumer of electricity [2]. This sector is located mainly in the major cities; however rural areas need energy supply for rural activities. Buildings are generally one of the main energy consumers in the urban context. Globally, buildings are responsible for approximately $40 \%$ of the total world's annual energy consumption [3]. Most of this energy is for the provision of lighting, heating, cooling, and air conditioning [4]. In Portugal, the building sector has the second highest growth rate of energy consumption, immediately after the transport sector [3].

In order to increase overall efficiency in the energy system, it would be helpful if part of the energy supply were generated at or near the points of demand. In this context, once microgeneration systems are capable of contributing with around $40 \%$ of energy demand, they can play an important role in decreasing the amount needed from big centralized fossil fired power plants and consequently reducing carbon emissions [5]. 
The technologies applied in small scale energy generation can generate electricity or heat and in some cases both. Small scale technologies for electricity production make use of microwind turbines, PV, and microhydro systems.

PV generation connected to the utility grid has gained growing attention, given the rapid increase in the world's demand for clean energy [6]. Microgeneration technologies, particularly PV installations, have a huge potential to be used in urban and rural environments, not only to satisfy demand and provide decentralized production, but also to help reduce fossil fuel dependency and reduce emissions [7]. In addition to the known environmental benefits, the impact of urban and rural renewable energy (RE) generation upon the occupant of a building or home can provide further impetus towards justifying its use and added costs [8].

The technology that uses the sun as an energy source presents direct and indirect benefits [9]. The direct benefits include sustainable electrical power generation and financial savings on the part of the producer and consumer. Indirect benefits include self-sustaining buildings, increased awareness about global warming, and about technical issues, such as the advantage of generating energy near the place of consumption and subsequent strengthening of the grid, and the capability of reinforcing the grid during periods of high energy demand.

This work results from a consultation made by the University of Beira Interior to two micro-PV generation private facilities connected to the grid with the objective of investigating their energy quality. We review work done in the field of solar microgeneration, present and compare the data from two different facilities with PV electricity generation, and discuss their behaviour. Section 2 presents the most important aspects of the case study PV facilities. Section 3 defines the methodologies and the analysis. Section 4 addresses the results, illustrated with graphical information about voltage fluctuation, frequency, and harmonics and discusses the results of the statistical analysis and Section 5 concludes the paper.

\section{Characteristics of Photovoltaic Generation}

Electrification of rural areas has always been a challenge, but PV systems can have a significant impact on rural development [10]. PV generation models are being applied all over the world [11-13], helping people living in rural areas to increase their participation in modern labor markets [14], reducing their expenses [15], and in some cases increasing schooling levels [16].

An advanced PV generation system should involve characteristics such as high efficiency, flexible control, safety, high reliability, and excellent scalability, wherein overall efficiency is the most important parameter to optimize cost reduction [6]. Scalability is related to the capacity of accommodating growth in the grid. Reliability, in contrast, refers to the consistency of electrical energy and its availability to meet peak demand. The energy's consistence has to be guaranteed as well as power quality, a very important issue contributing to improving the quality of electrical components. The main problems regarding power quality are high harmonic distortions and voltage levels [17].

A local decentralized RE approach could be a solution to smooth the impacts of energy failures, as well as to allow peak shaving during busy energy supply periods. Governments are fostering the use of these technologies by using a feed-in electrical tariff, but its implementation is strongly dependent on economical/financial aspects [18]. The main objective and challenge of RE integration in decentralized locations are the incorporation of technically and economically viable systems in collecting and processing RE sources. Decentralized energy production, that is, distributed generation (DG), has high economic potential. These systems are expected to play a significant role in local power generation of European countries, particularly in Southern Europe [19]. A DG agglomeration forms a microgrid (MG), term first used in 2001 [20], which can function either connected to the grid or in isolation. The impact upon power quality relies on the MG connection to the DG [21].

Microgrids comprise low voltage (LV) distribution systems [22] together with distributed energy sources and storage devices as energy capacitors and batteries [23], which have nonlinear electronic devices. These cause considerable harmonic pollution in power supply systems, having severe undesirable influences on system components by changing parameters of grid impedance, distorting the fundamental voltage and current waveforms, influencing the availability of short circuit power, overheating the transformers and cables, and causing resonance and false operation of protection devices [17]. Once it becomes prominent, it will decrease power quality and increase unexpected maintenance processes and expenses [5].

The application of PV systems in power systems as a safe and clean source of energy from the sun can be divided into two main fields, namely, stand-alone and gridconnected applications [24]. Microgeneration technologies can be employed in a number of ways that imply different roles for energy consumers and companies. PV microgeneration appears to be highly appreciated, once it has very low manufacturing costs leading to affordable solar electricity [25], as well as an improved module efficiency, cost reduction, and increased productivity [26].

The two grid-connected systems under study are located in a countryside region of Portugal, with a peak installed power of $4.14 \mathrm{kWp}$ and $3.87 \mathrm{kWp}$, for the first and second facility, respectively. They are covered by a special regime, under Decree Law number 363/2007 [27], whereby all holders of a contract for electricity consumption at low voltage can produce and sell electricity to the grid for 15 years. This subsidized regime is applied to microunits with a capacity limited to $50 \%$ of the contracted power and not more than $3.68 \mathrm{~kW}$ of delivered peak power, using the following energy sources: solar, wind, hydro, and biomass cogeneration [27].

The first facility is constituted by 18 solar panels type Sanyo HIP-230HDE1 (HIT). The panels are installed in a twoaxis tracking system, DEGER3000NT. The panel strings (2 modules in parallel and 9 panels per module) are connected to the grid through a SMA Solar Sunny Boy SB 3800/V converter. 


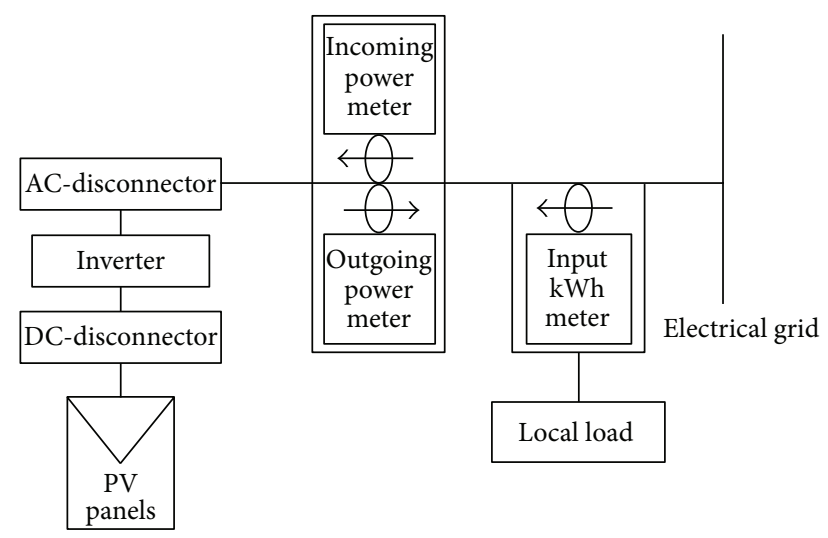

FiguRE 1: System block diagram.

The second facility is constituted by 18 solar panels type Sanyo HIP-215NKHE1. The panels are installed in a fixed structure without tracking. The panel strings ( 2 modules in parallel and 9 panels per module) are connected to the grid through a SMA Solar Sunny Boy SB 3300 converter.

Figure 1 shows the block diagram of both systems. The two facilities are located in a rural area, fed by a low voltage line derived from an aerial transformer, and have a similar structure.

\section{Methodologies and Analysis}

The monitoring of each referred facility started with the installation of a single phase voltage quality recorder Fluke VR1710 to collect data correspondent to the voltage evolution in an interval of time for posterior analysis of each facility's operational behavior and voltage quality. This device was capable of recording all the data analyzed in this paper by using an appropriate voltage probe. It was also capable of recording all values without gaps, obtaining the whole picture with one instrument that records both events and voltage. The RMS average, minimum, and maximum values can be recorded from $1 / 4$ of a cycle. For this work, the values were recorded from $1 / 2$ of a cycle. This allows detection of all drops in voltage with no interruptions. In these systems, the analyses were centered in two different power quality factors: the fluctuation of the PV system output power and the voltage harmonic distortion.

Both facilities are similar; however the first has a tracking system and a little bit more power than the second, thus injecting more power into the network. This work will show that the second facility has more problems, arising from the amount of injected power. Therefore, a major task of this work was the comparison of the operating mode and the power quality of both facilities. The former is particularly relevant in rural facilities with weakly meshed distribution systems and is usually considered a serious problem in PV systems, due to severe operational problems to the utility network caused by variations in solar irradiance (depending on climate conditions or panel placement), as power oscillates in lines, under and over loading, causing critical voltage fluctuations and voltage flickers [28]. The latter factor, also

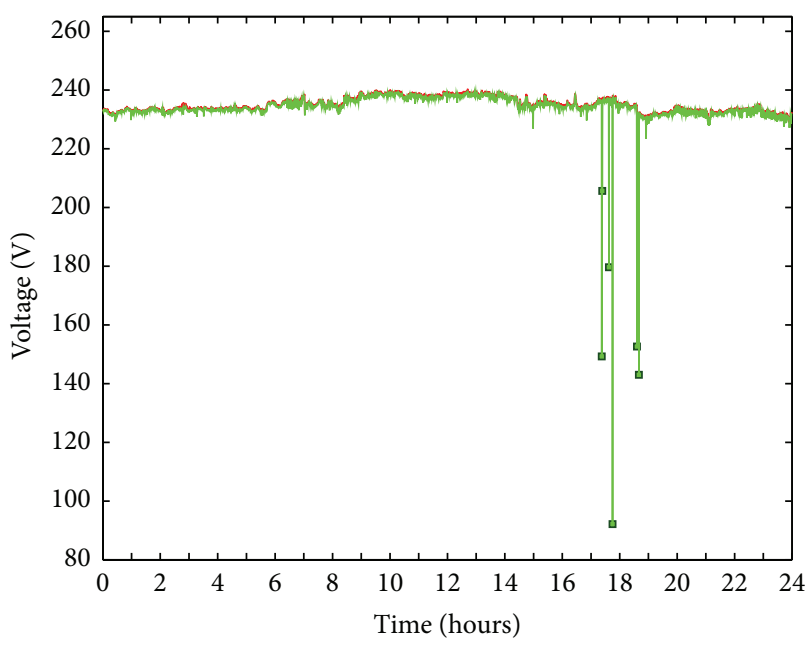

FIGURE 2: Voltage values registered during one day in the first facility. The green line represents the minimum voltages, where the green big dots are the voltage dips, the black line represents the medium voltages, and the red line represents the maximum voltages.

a serious issue in PV systems, may occur due to the use of power inverters and decreases the reliability of power systems [29].

The equipment was configured to start data recording at the same time as the team visit and lasted seven days. The data were recorded in the same week during the summer, but in different locations. The voltage values were registered every five seconds. For this work, the day with most solar irradiance was chosen as the most representative for illustration purposes. In total, 17280 values of voltage and frequency and 144 values of harmonics were collected from both facilities. The voltage dips were defined as values equal or lower to $207 \mathrm{~V}$ and the voltage swells were defined as values equal or higher to $253 \mathrm{~V}$, for a $230 \mathrm{~V}$ rated voltage. After the recording period, the data were transferred to a computer to be analyzed, as described in the next section.

\section{Results and Discussion}

The acquired data were organized into two sets of values, one for data recorded in each facility. For illustration purposes, we rely on data recorded during a single day in each facility.

4.1. Voltage Profile. Figure 2 represents the voltage profile for the first facility during the third day of data recording. Dips are only observed in the evening: seven dips were registered and the largest case presents a very low value of voltage, below $100 \mathrm{~V}$ and nearly $60 \%$ lower than the rated voltage $(230 \mathrm{~V})$, the limit imposed by the European Standard EN 50160 [30]. In this facility, no swells were recorded during the seven days.

Figure 3 represents the voltage profile for the second facility observed during the second day of data recording. During the daylight period, swells occurred frequently, with 9634 swells registered, the largest case corresponding to a maximum voltage value of $260.75 \mathrm{~V}$, which is $3.06 \%$ higher 


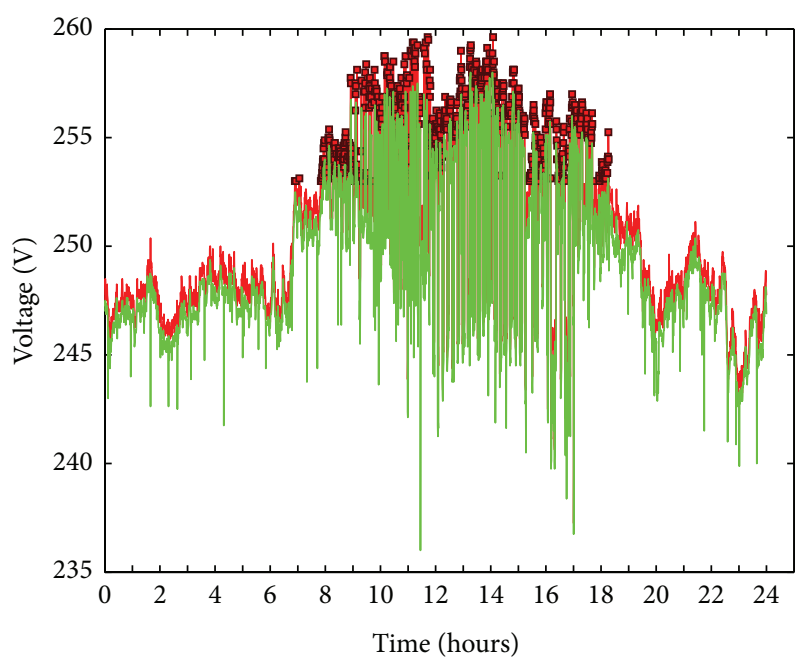

FIGURE 3: Voltage values registered during one day in the second facility. The green line represents the minimum voltages, the black line represents the medium voltages, and the red line represents the maximum voltages.

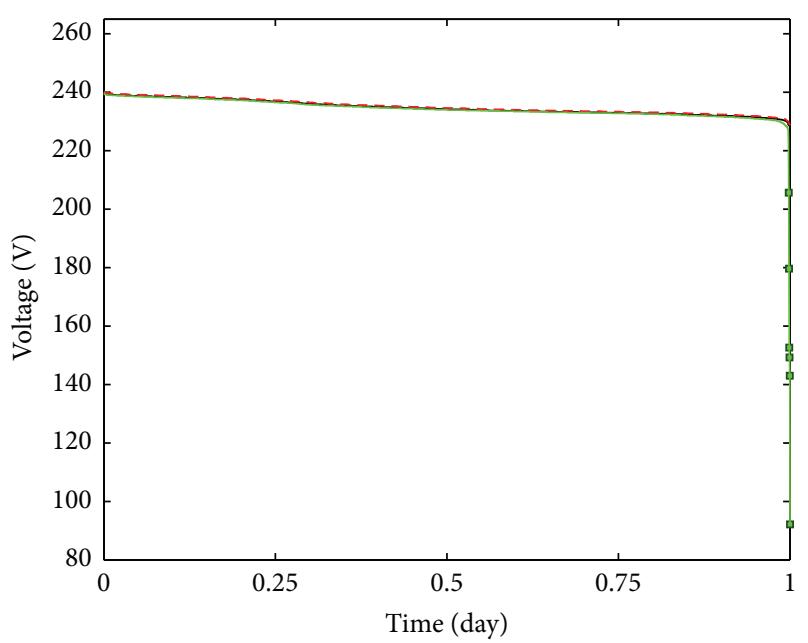

FIGURE 4: Voltages of the first facility during the recording period with the values sorted in descending order.

than the limit $(253 \mathrm{~V})$ imposed by the standard [30]. In this facility, no dips were recorded during the seven days.

The operational behaviour of both facilities is clearly different. The first facility never exceeded the maximum limit imposed by the standard [30]; however it registered a few dips, around 6 p.m., which corresponded to a fault in the network. The second facility revealed operational problems during working hours. From the obtained results one can conclude that the most influential parameter in the operation of the PV system is voltage fluctuation, particularly overvoltage exceeding the standard limit. As a consequence the inverter will automatically connect and disconnect from the grid.

Figure 4 shows the voltage values of the first facility organized in descending order. It is possible to see that

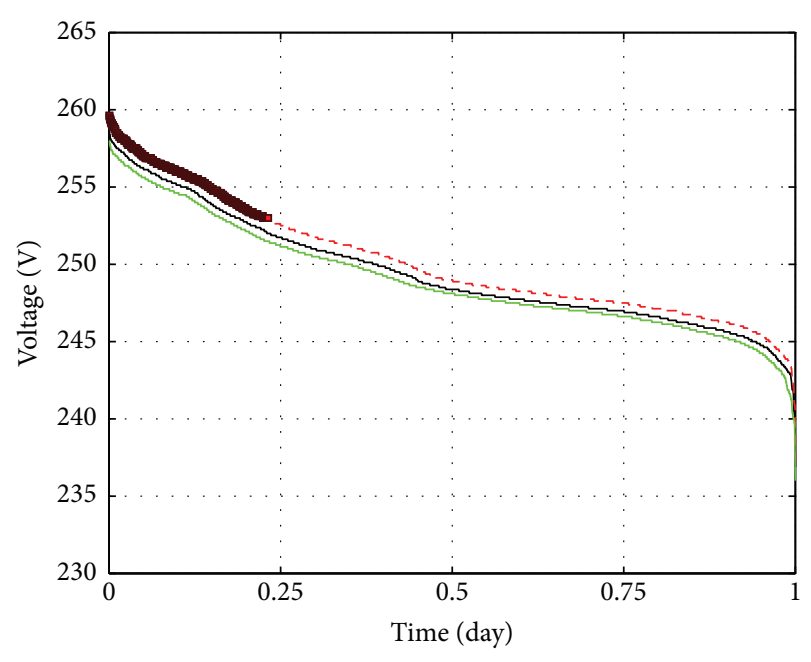

FIGURE 5: Voltages of the second facility during the recording period with the values sorted in descending order.

the voltage values stay between the limits during most of the day, except the few dips around 6 p.m.

Figure 5 shows the results obtained from the second facility with the voltage values sorted in descending order. It is possible to see that during more or less a quarter of the day the maximum voltages are above the upper limit.

The first facility exhibited voltage values between $220 \mathrm{~V}$ and $240 \mathrm{~V}$, a very tiny interval, with no values outside the boundaries, which is a very regular behaviour for the voltage profile. Thus, the corresponding PV system is operating properly. The second facility exhibited quite a different behaviour: during a quarter of the day, all the voltage values were above $253 \mathrm{~V}$ (upper limit imposed by the standard), including the minimum, medium, and maximum values. Thus, the corresponding PV system operation is inadequate.

4.2. Harmonic Distortion and Harmonics. Figure 6 presents the harmonic distortion for the third day of data recording (same as above) in the first facility. The THD and the 3rd, 5th, and 7 th harmonic distortions are clearly below the allowed limits: $8 \%, 5 \%$, and $6 \%$, respectively, as stated in [30].

Figure 7 presents the harmonic distortions for the second day of data recording (same as above) in the second facility. The THD and the 3rd, 5th, and 7th harmonic distortions are clearly below the allowed limits: $8 \%, 5 \%, 6 \%, 0.2 \%$, and $0.1 \%$ for the remaining harmonic order, respectively, as stated in [30].

4.3. Frequency. Figure 8 shows the frequency profile for both facilities. The deviation is clearly below the absolute value of $0.5 \mathrm{~Hz}$ imposed by the standards [30]. It is possible to see a consistent behaviour, independent of the voltages profiles. The voltage profile is imposed by the inverter of the PV system, rather than any network malfunction.

4.4. Statistical Analysis. Statistical analysis of the voltage values was performed to describe differences in severity of the fluctuations in the grid voltage of both facilities, considering 


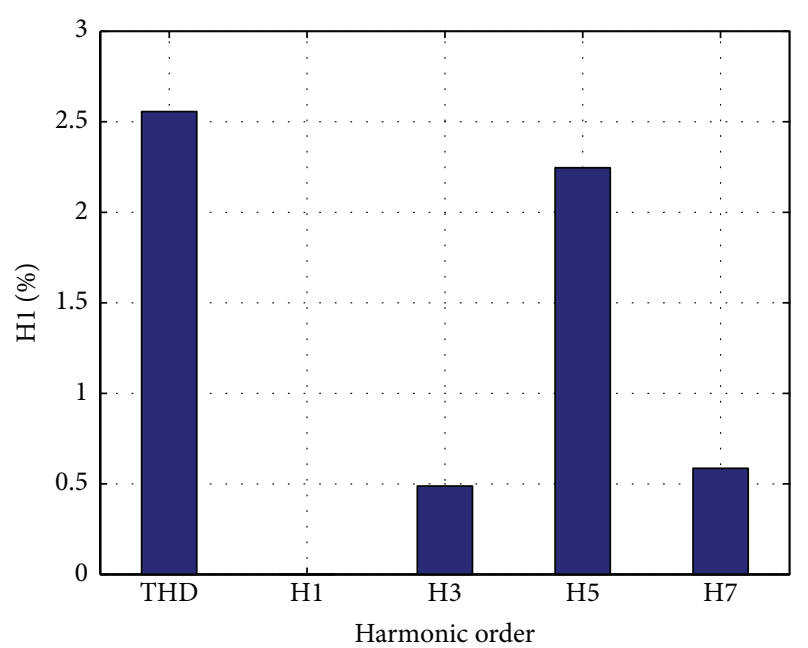

FIGURE 6: Total harmonic distortions registered during the day in the first facility.

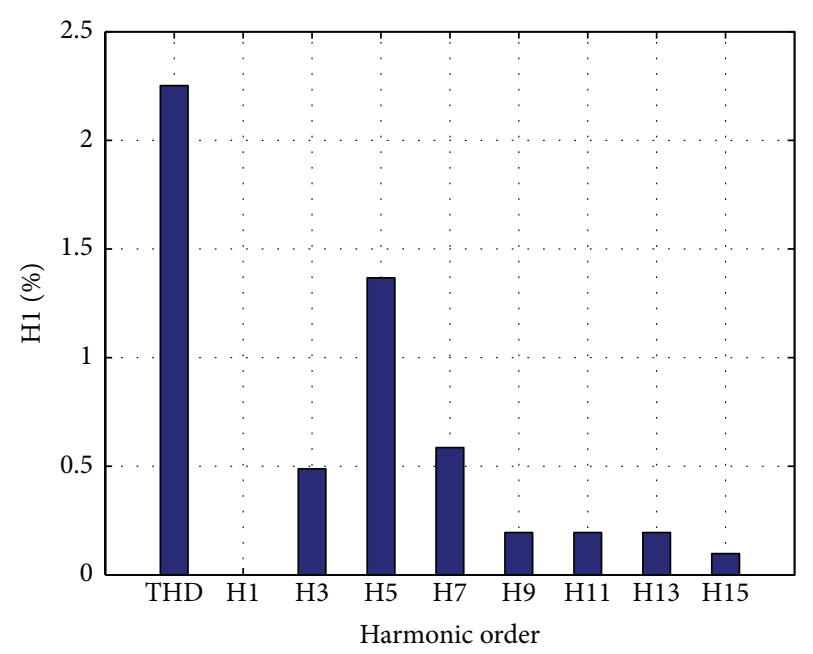

FIGURE 7: Total harmonic distortions registered during the day in the second facility.

all the data recorded. The histograms with the minimum, medium, and maximum voltage values were obtained, representing the number of occurrences and the duration of dips and swells in each range of voltages. Figure 9 shows the obtained histograms for the first facility. The minimum voltage had a mean value of $234.44 \mathrm{~V}$ with a standard deviation of $2.894 \mathrm{~V}$. The $95 \%$ percentile was $238.5 \mathrm{~V}$ indicating that $5 \%$ of the voltages were above $3.7 \%$ of the rated voltage. Also, $99.97 \%$ of the voltage values were within the $90 \%-110 \%$ range; thus about $0.03 \%$ of them were outside the standard limits. The medium voltage had a mean value of $234.73 \mathrm{~V}$ with a standard deviation of $2.359 \mathrm{~V}$. The $95 \%$ percentile was $238.8 \mathrm{~V}$, indicating that $5 \%$ of the voltages were above $3.83 \%$ of the rated voltage. Also, $100 \%$ of the voltage values were within the $90 \%-110 \%$ range. The maximum voltage had a mean value of $235.15 \mathrm{~V}$ with a standard deviation of $2.365 \mathrm{~V}$.
The $95 \%$ percentile was $239.1 \mathrm{~V}$ indicating that more than $5 \%$ of the voltages were above $3.96 \%$ of the rated voltage. Once again, $100 \%$ of the voltage values were within the $90 \%-110 \%$ range.

Figure 10 represents the statistical analysis for the minimum, medium, and maximum voltage values, respectively, for the second facility. The minimum voltage had a mean value of $249.03 \mathrm{~V}$ with a standard deviation of $3.504 \mathrm{~V}$. The $95 \%$ percentile was $255.6 \mathrm{~V}$ indicating that $5 \%$ of the voltages were above $11.3 \%$ of the rated voltage. Also, $84.12 \%$ of the voltage values were within the $90 \%-110 \%$ range; thus about $15.88 \%$ of them were outside the standard limits. The medium voltage values had a mean value of $249.48 \mathrm{~V}$ with a standard deviation of $3.082 \mathrm{~V}$. The $95 \%$ percentile was $256.3 \mathrm{~V}$ indicating that $5 \%$ of the voltages were above $11.43 \%$ of the rated voltage. Also, $81.11 \%$ of the voltage values were within the $90 \%-110 \%$ range; thus about $18.89 \%$ of them were outside the standard limits. The maximum voltage had a mean value of $250.16 \mathrm{~V}$ with a standard deviation of $3.674 \mathrm{~V}$. The $95 \%$ percentile was $257 \mathrm{~V}$ indicating that more than $5 \%$ of the voltages were above about $11.74 \%$ of the rated voltage. Also, $79.09 \%$ of the voltage values were within the $90 \%-110 \%$ range; thus about $20.91 \%$ of them were outside the standard limits.

The voltage dips and voltage swells during the day of most solar irradiance are represented in Figures 11 and 12, for the first and second facilities, respectively. The vertical axis represents the voltage level of the dip or swell event and the duration of the event is marked on the horizontal axis. The green circles represent the voltage dips and the red circles represent the voltage swells. The combination of the level and duration of the occurrence indicates the severity of the dip or swell event.

Figure 11 shows that the first facility operates with voltage levels within the standard limits. Only a very small number of dips (7 dips during a full day) occurred, with no swells.

In Figure 12 it is possible to observe that, for the second facility, the swells are all near $110 \%$ of the rated voltage due to inverter operation. Since there is sufficient solar irradiation, the PV inverter is capable of providing power. When the rural low voltage grid has a suitable voltage and frequency, the inverter connects to the grid at its AC terminals, synchronization occurs, and the inverter starts supplying power. Once this occurs, the voltage rises and reaches the voltage limit imposed by the inverter in order to stay connected. The voltage rises because there are not enough loads connected to the grid near the injection point. This operation is constantly repeated causing voltage fluctuations (the voltage starts to rise when the inverter connects to the grid and drops when the inverter disconnects).

One difference between the two facilities is the duration of each event. In the first facility, the longest registered event lasted $1.2 \mathrm{sec}$, and in the other facility, generally with longer events, the longest registered event lasted $330 \mathrm{sec}$.

A second difference is that only voltage dips were registered in the first facility, while the other facility only presented voltage swells. This difference is a consequence of the inadequate PV system operation which forces the inverter to automatically connect and disconnect from the grid. 


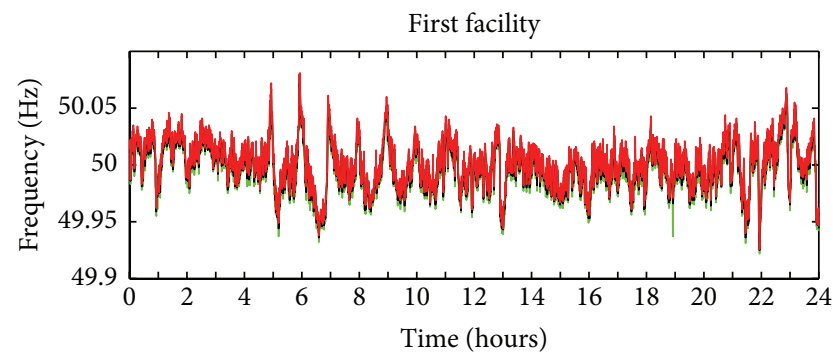

(a)

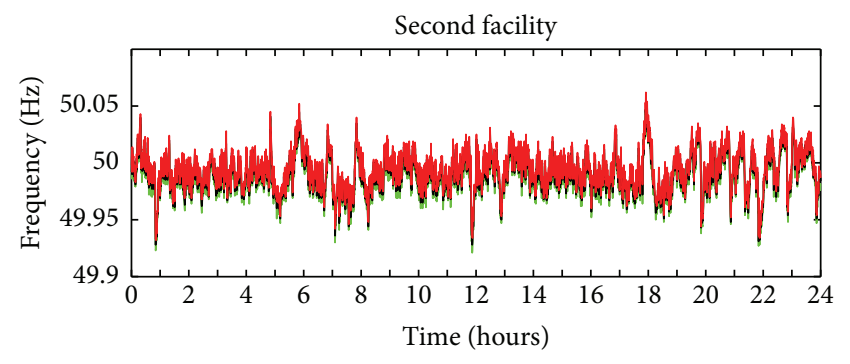

(b)

Figure 8: Frequency evolution in both facilities. The green line represents the minimum voltages, the black line represents the medium voltages, and the red line represents the maximum voltages.

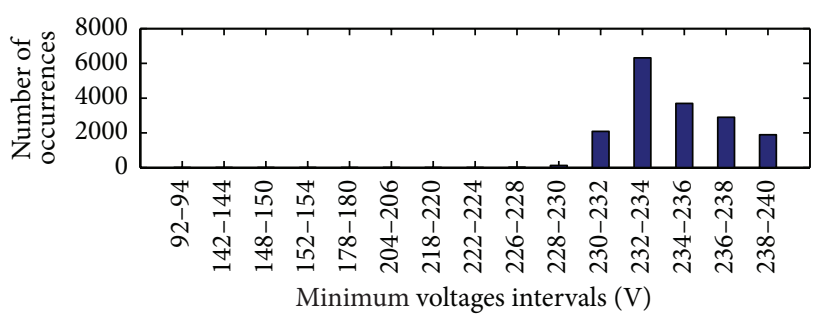

(a)

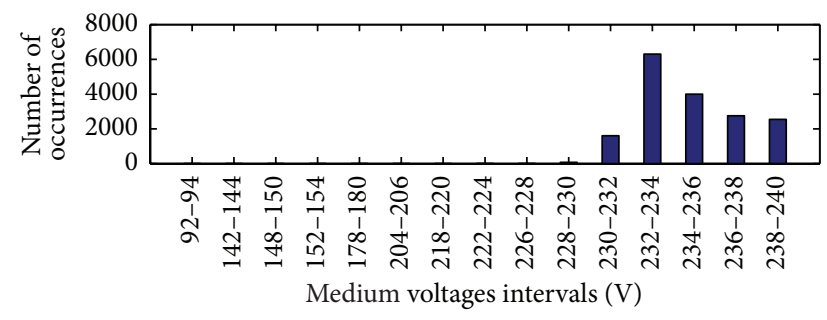

(b)

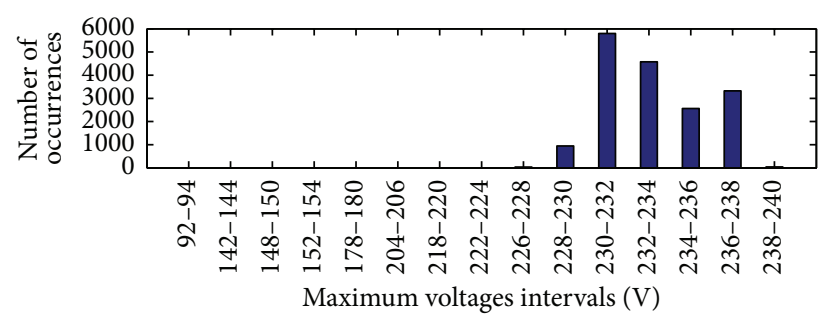

(c)

FIGURE 9: Statistical voltage distributions in the first facility.

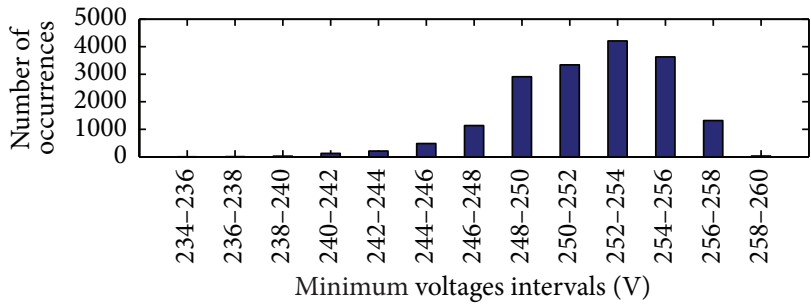

(a)

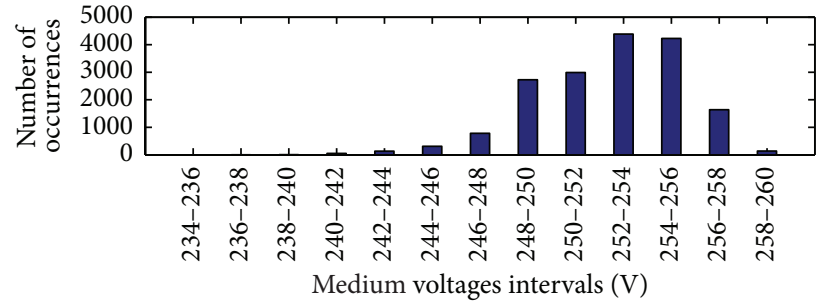

(b)

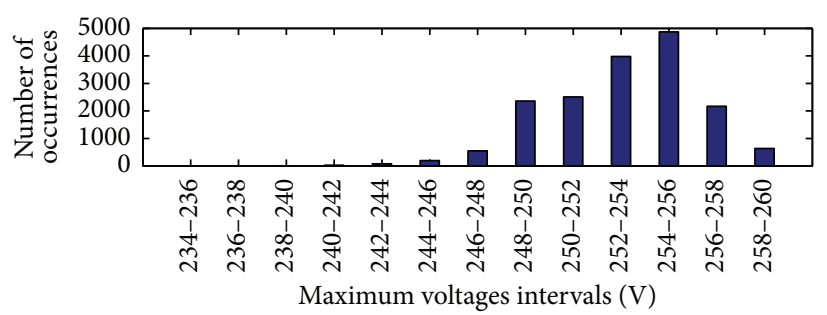

(c)

FIGURE 10: Statistical voltage distribution in the second facility. 


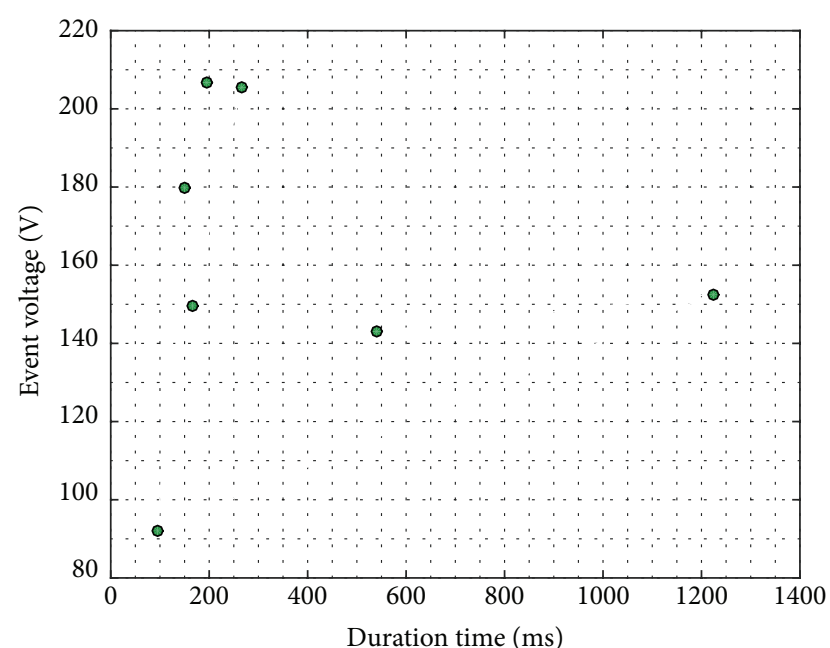

FIGURE 11: Occurrences of voltage dips and voltage swells for the first facility. The green circles represent the minimum values below the lower limits and the red circles represent the maximum values above the upper limits imposed by the standard.

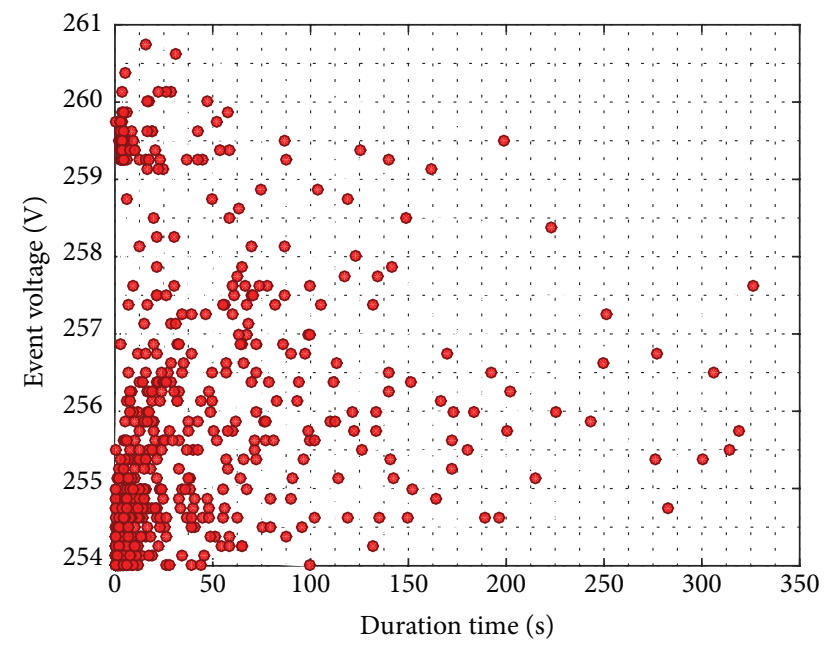

FIGURE 12: Occurrences of voltage dips and swells for the second facility. The green circles represent the minimum values below the lower limits and the red circles represent the maximum values above the upper limits imposed by the standard.

\section{Conclusions}

The presence of grid-connected PV generators always produces a rise in the voltage profile due to the decrease in the total load. This rise may cause overvoltage and, of course, this is a hindrance to the penetration of DG units.

This paper presents the results of a power quality analysis of two real PV generation facilities connected to a rural LV grid. The voltage fluctuations and the voltage harmonic content were observed and described statistically.

The first facility showed very satisfactory results compared to the second one. The infrastructure did not present maximum voltage values exceeding the upper limit, and minimum values below the lower limit were scarce and were resulting from the network operation. We can conclude that this facility appears to be economically viable for microgeneration.

The second facility, in contrast, exhibited a considerable percentage of voltages exceeding the standard limit and the inverter technical maximum voltage limit. In particular, for the maximum voltage values, when comparing the percentage of the minimum and the maximum voltages outside the $90 \%-110 \%$ range, it can be concluded that the limits were violated mainly due to voltages above $110 \%$ of the rated voltage. The number of times this occurs contributes to decreasing the amount of energy delivered to the grid (the inverter only stays connected during a small period of time during the day), implying losses in revenue and making this PV facility economically unviable. In addition, the severity of the voltage fluctuations imposed on the grid, near the injection point, results in increased voltage pollution, that is, decreasing in voltage power quality.

\section{Conflict of Interests}

The authors declare that there is no conflict of interests regarding the publication of this paper.

\section{References}

[1] DGEG-Direção Geral de Energia e Geologia, http://www .dgeg.pt/.

[2] C. Camus and E. Eusébio, "Micro-generation evaluation of the zero emissions technologies in the Portuguese market," in Proceedings of the 5th International Conference on the European Electricity Market (EEM '08), pp. 1-6, IEEE, Lisboa, Portugal, May 2008.

[3] A. Duarte, D. Coelho, and N. Tomás, "Photovoltaic integration in buildings. A case study in Portugal," in Proceedings of the International Conference on Renewable Energies Sources, pp. 119-123, Sousse, Tunisia, 2010.

[4] A. M. Omer, "Energy, environment and sustainable development," Renewable and Sustainable Energy Reviews, vol. 12, no. 9, pp. 2265-2300, 2008.

[5] J. Watson, R. Sauter, B. Bahaj, P. James, L. Myers, and R. Wing, "Domestic micro-generation: economic, regulatory and policy issues for the UK," Energy Policy, vol. 36, no. 8, pp. 3085-3096, 2008.

[6] L. Zhang, K. Sun, Y. Xing, L. Feng, and H. Ge, "A modular gridconnected photovoltaic generation system based on DC bus," IEEE Transactions on Power Electronics, vol. 26, no. 2, pp. $523-$ 531, 2011.

[7] A. S. Bahaj and P. A. B. James, "Urban energy generation: the added value of photovoltaics in social housing," Renewable and Sustainable Energy Reviews, vol. 11, no. 9, pp. 2121-2136, 2007.

[8] A. Chaurey and T. C. Kandpal, "Assessment and evaluation of PV based decentralized rural electrification: an overview," Renewable and Sustainable Energy Reviews, vol. 14, no. 8, pp. 2266-2278, 2010.

[9] A. S. Bahaj and P. A. B. James, "Direct and indirect benefits of grid connected photovoltaics in low energy social housing," in Proceedings of the 8th World Renewable Energy Congress (WREC '04), 2004. 
[10] B. Van Campen, D. Guidi, and G. Best, "Solar photovoltaics for sustainable agriculture and rural development," in Rural Development, FAO Publication, 2000.

[11] S. Karekezi and W. Kithyoma, "Renewable energy strategies for rural Africa: is a PV-led renewable energy strategy the right approach for providing modern energy to the rural poor to subSaharan Africa?” Energy Policy, vol. 30, no. 11-12, pp. 1071-1086, 2002.

[12] K. Q. Nguyen, "Alternatives to grid extension for rural electrification: decentralized renewable energy technologies in Vietnam," Energy Policy, vol. 35, no. 4, pp. 2579-2589, 2007.

[13] C. E. C. Nogueira and H. H. Zürn, "Modelo de dimensionamento otimizado para sistemas energéticos renováveis em ambientes rurais," Engenharia Agrícola, vol. 25, no. 2, pp. 341348, 2005.

[14] T. Dinkelman, "The effects of rural electrification on employment: new evidence from South Africa," American Economic Review, vol. 101, no. 7, pp. 3078-3108, 2011.

[15] A. A. Lahimer, M. A. Alghoul, F. Yousif, T. M. Razykov, N. Amin, and K. Sopian, "Research and development aspects on decentralized electrification options for rural household," Renewable and Sustainable Energy Reviews, vol. 24, pp. 314-324, 2013.

[16] F. S. Javadi, B. Rismanchi, M. Sarraf et al., "Global policy of rural electrification," Renewable and Sustainable Energy Reviews, vol. 19, pp. 402-416, 2013.

[17] J. F. G. Cobben, W. L. Kling, and J. M. A. Myrzik, "Power quality aspects of a future micro grid," in Proceedings of the International Conference on Future Power Systems, pp. 1-5, IEEE, Amsterdam, Netherlands, November 2005.

[18] A. Roque, N. Fontes, J. Maia, C. Casimiro, and D. M. Sousa, "Economic aspects of a domestic micro-generation system," in Proceedings of the 6th International Conference on the European Energy Market (EEM '09), pp. 1-6, IEEE, Leuven, Belgium, May 2009.

[19] N. Hatziargyriou, "Microgrids-large scale integration of micro-generation to low voltage grids," in Proceedings of the CIGRÉ Session, Paper no. C6-309, Paris, France, 2006.

[20] B. Lasseter, "Microgrids [distributed power generation]," in Proceedings of the IEEE Power Engineering Society Winter Meeting, vol. 1, pp. 146-149, 2001.

[21] C. Natesan, S. K. Ajithan, P. Palani, and P. Kandhasamy, "Survey on microgrid: power quality improvement techniques," ISRN Renewable Energy, vol. 2014, Article ID 342019, 7 pages, 2014.

[22] M. Klatt, A. Dorado, J. Meyer, P. Schegner, J. Backes, and R. $\mathrm{Li}$, "Power quality aspects of rural grids with high penetration of microgeneration, mainly PV-installations," in Proceedings of the 21st International Conference on Electricity Distribution, no. 0273, pp. 1-4, 2011.

[23] P. M. Costa and M. A. Matos, "Economic analysis of microgrids including reliability aspects," in Proceedings of the 9th International Conference on Probabilistic Methods Applied to Power Systems (PMAPS '06), pp. 1-8, June 2006.

[24] M. Farhoodnea, A. Mohamed, H. Shareef, and H. Zayandehroodi, "Power quality impact of grid-connected photovoltaic generation system in distribution networks," in Proceedings of the IEEE Student Conference on Research and Development (SCOReD' 12), pp. 1-6, December 2012.

[25] B. Azzopardi, C. J. M. Emmott, A. Urbina, F. C. Krebs, J. Mutale, and J. Nelson, "Economic assessment of solar electricity production from organic-based photovoltaic modules in a domestic environment," Energy and Environmental Science, vol. 4, no. 10, pp. 3741-3753, 2011.

[26] Z. Li, F. Boyle, and A. Reynolds, "Domestic application of solar PV systems in Ireland: the reality of their economic viability," Energy, vol. 36, no. 10, pp. 5865-5876, 2011.

[27] Portuguese Decree-Law, No. 363/2007 of 2nd November, pp. 222-229, 2012.

[28] O. M. Toledo, D. O. Filho, and A. S. A. C. Diniz, "Distributed photovoltaic generation and energy storage systems: a review," Renewable and Sustainable Energy Reviews, vol. 14, no. 1, pp. 506-511, 2010.

[29] M. Farhoodnea, A. Mohamed, H. Shareef, and H. Zayandehroodi, "An enhanced method for contribution assessment of utility and customer harmonic distortions in radial and weakly meshed distribution systems," International Journal of Electrical Power and Energy Systems, vol. 43, no. 1, pp. 222-229, 2012.

[30] CENELEC, "Voltage characteristics of electricity supplied by public distribution networks," European Standard EN 50160, CENELEC, 2007. 

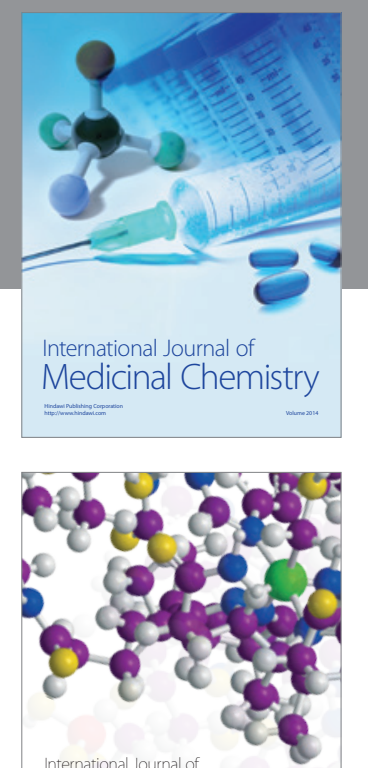

\section{Carbohydrate} Chemistry

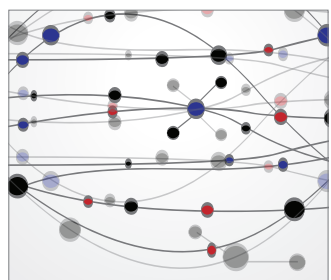

The Scientific World Journal
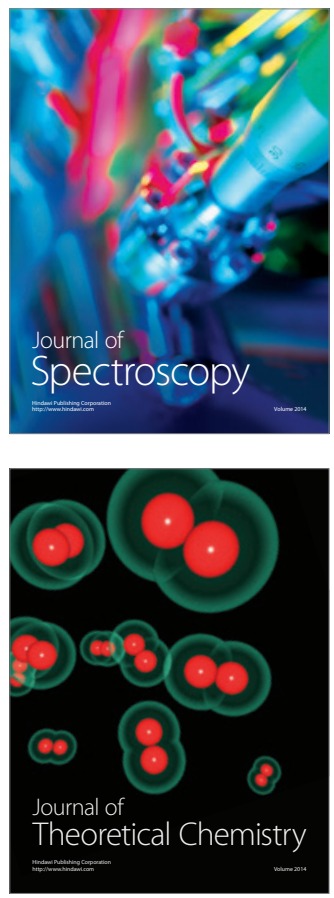
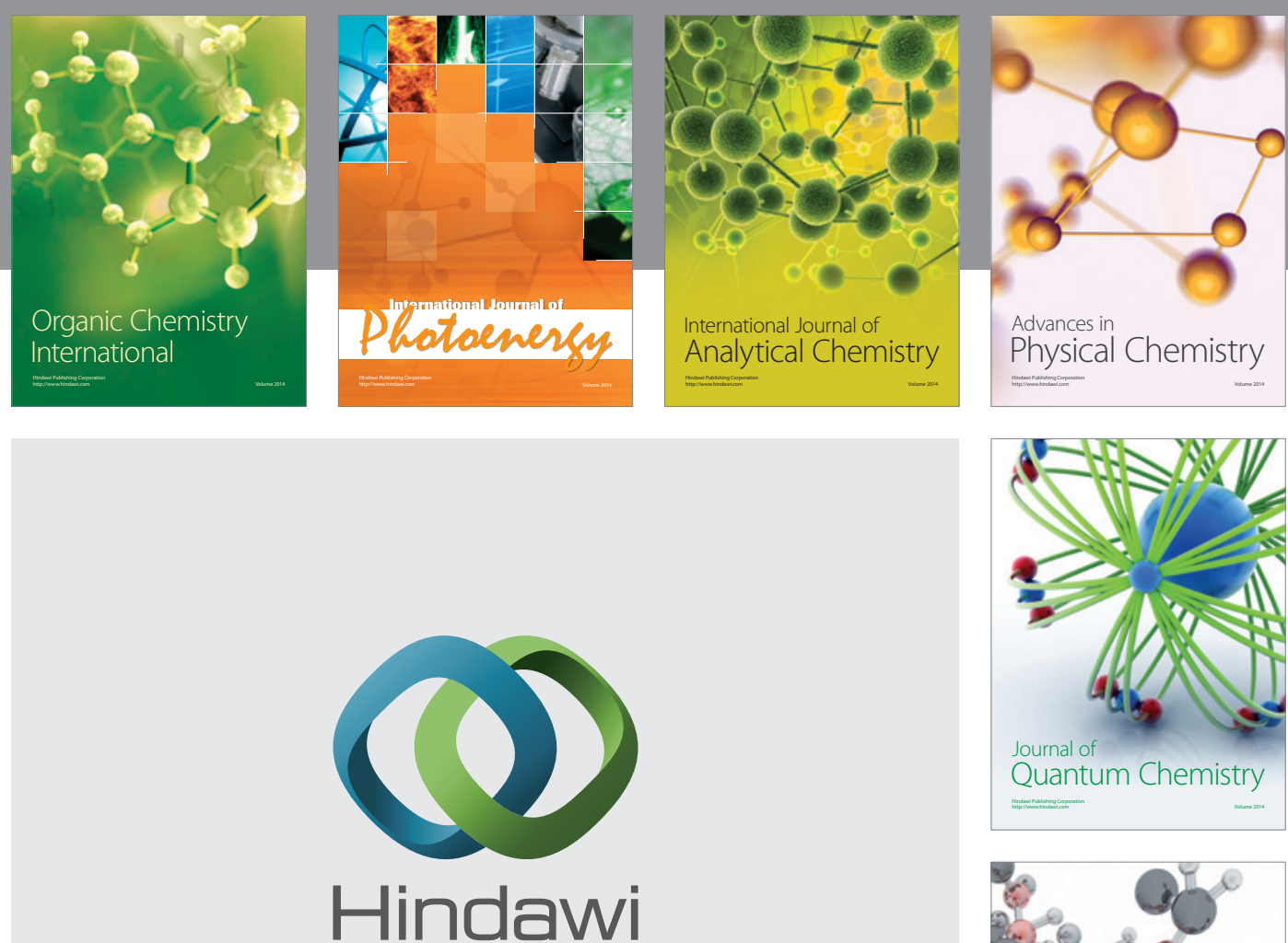

Submit your manuscripts at

http://www.hindawi.com

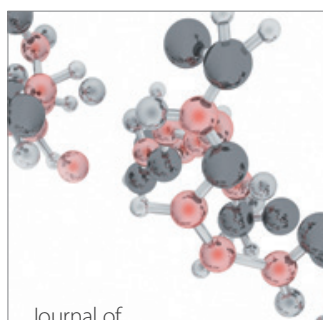

Analytical Methods

in Chemistry

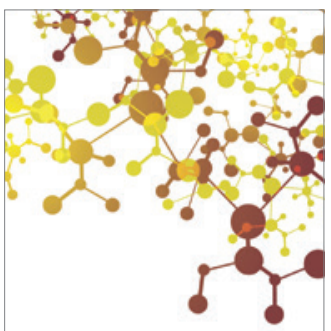

Journal of

Applied Chemistry

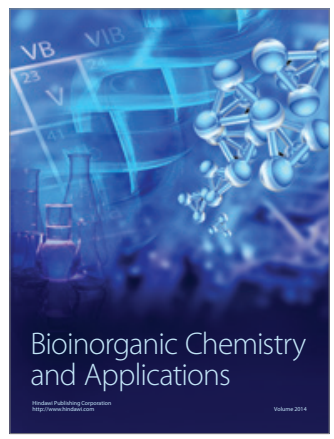

Inorganic Chemistry
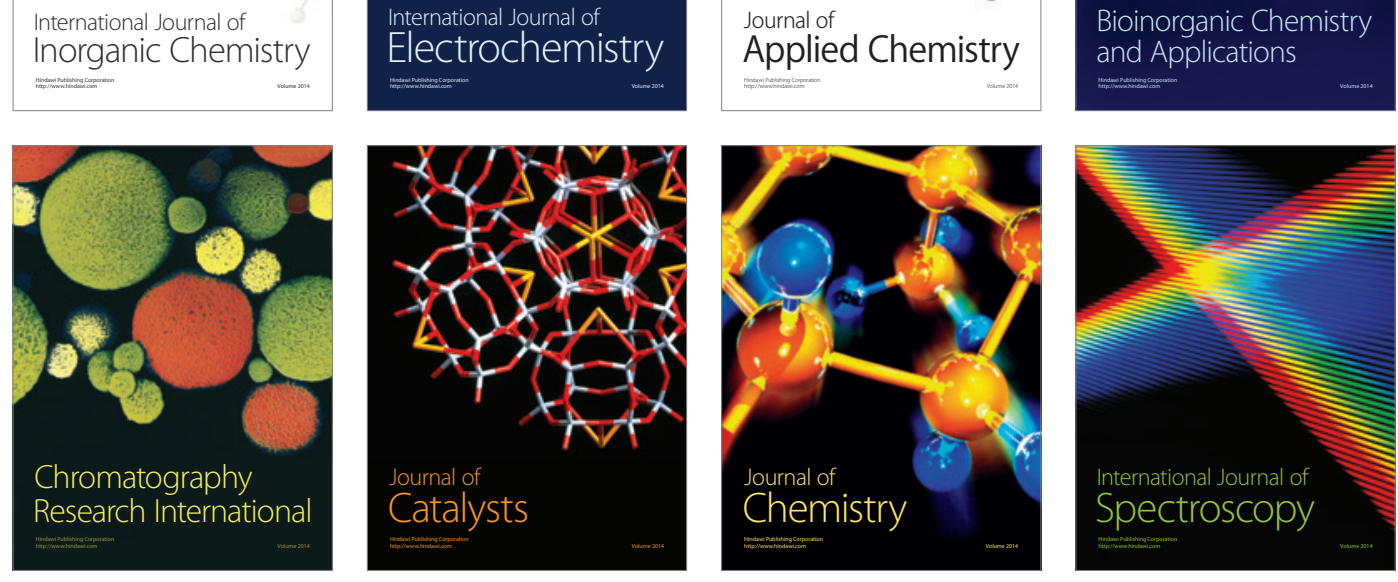